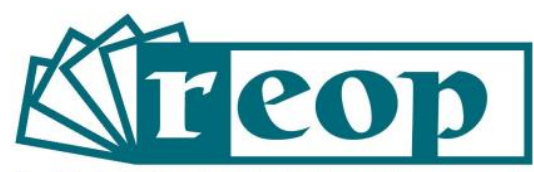

Revista Española de Orientación y Psicopedagogía

\title{
LOS DISPOSITIVOS MÓVILES EN EL HOGAR. INTERÉS FORMATIVO DE LAS FAMILIAS ESPAÑOLAS ${ }^{1}$
}

\section{MOBILE DEVICES AT HOME. FORMATIVE INTEREST OF SPANISH FAMILIES}

\author{
Antonia Ramírez-García² \\ Universidad de Córdoba. Facultad de Ciencias de la Educación. Departamento de Educación. \\ Córdoba, España \\ Irina Salcines-Talledo \\ Natalia González-Fernández \\ Universidad de Cantabria. Facultad de Educación. Departamento de Educación. Santander, España
}

\section{RESUMEN}

La sociedad de las primeras décadas del siglo XXI ha comenzado a denominarse de diferentes modos, pero todos ellos refiriéndose a la tecnología, la digitalización, las pantallas o la

\footnotetext{
1 Fuente de financiación: Proyecto $++\mathrm{D}+\mathrm{I}$ Coordinado "Competencias mediáticas de la ciudadanía en medios digitales emergentes (Smartphones y Tablets): practicas innovadoras y estrategias educomunicativas en contextos múltiples" (EDU2015-64015-C3-1-R) (MINECO/FEDER), y de la "Red de Educación Mediática" del Programa Estatal de Investigación Científica-Técnica de Excelencia, Subprograma Estatal de Generación de Conocimiento (EDU201681772-REDT), financiados por el Fondo Europeo de Desarrollo. Este trabajo también se enmarca en 'Alfamed' (Red Euroamericana de Investigadores), con el apoyo del Proyecto I+D "Youtubers e instagrammers: La competencia mediática en los prosumidores emergentes" (RTI2018-093303-B-I00), financiado por la Agencia Estatal de Investigación del Ministerio de Ciencia, Innovación y Universidades de España y el Fondo Europeo de Desarrollo Regional (FEDER). ${ }^{2}$ Correspondencia: Antonia Ramírez-García. Dirección postal completa: Universidad de Córdoba. Facultad de Ciencias de la Educación. Departamento de Educación Avda. San Alberto Magno s/n. 14071. Córdoba. España. Teléfono: 957212600. Correo-e: a.ramirez@uco.es
} 
conectividad. La proliferación de la tecnología en diferentes ámbitos sociales, económicos, políticos o culturales también ha llegado a los hogares españoles a través de ordenadores, SmartTV o electrodomésticos inteligentes, pero, sin duda, mediante el acceso a Internet utilizando dispositivos móviles. El estudio que se presenta forma parte de una investigación de alcance nacional, en la que se analiza el uso de Smartphones y Tablets en los hogares españoles. La metodología empleada es básicamente cuantitativa y centrada en la implementación de un cuestionario diseñado ad hoc para la investigación, en este caso focalizado en la predisposición de las familias a la formación, la temática y el formato más adecuado para dicha información. La muestra participante ha sido de 1082 integrantes de unidades familiares de todas las Comunidades Autónomas. Los resultados muestran que las familias tienen un interés moderado en la formación sobre gestión de los dispositivos móviles con menores (55.7\%), la temática más aceptada por las familias es el aprendizaje de estrategias de seguimiento del uso de los dispositivos móviles, mientras que el formato más rechazado es el uso de documentos escritos o cursos presenciales. Estos resultados permiten proponer formación y orientación a las familias utilizando medios tecnológicos. En conclusión, la orientación encuentra en esta situación un campo de desarrollo y crecimiento nuevo en esta sociedad líquida.

Palabras clave: dispositivos móviles, familias, formación parental, orientación familiar, educación mediática.

\section{ABSTRACT}

The society of the first decades of the 21st century has begun to be called in different ways, but all of them referring to technology, digitalization, screens or connectivity. The proliferation of technology in different social, economic, political or cultural fields has also reached Spanish households through computers, SmartTV or smart appliances, but without a doubt, through Internet access using mobile devices. The study that is presented is part of a research of national scope, which analyzes the use of Smartphones and Tablets in Spanish households. The methodology used is basically quantitative and focused on the implementation of a questionnaire designed ad hoc for research, in this case focused on the predisposition of the families to the training, the theme and the format most suitable for that information. The participant sample has been 1082 members of families of all the Autonomous Communities. Results show that families have a moderate interest in training on the management of mobile devices with minors $(55.7 \%)$, the topic that is most accepted by families is learning strategies to follow the use of mobile devices in the families, while the format that is most rejected is the use of written documents or face-to-face training. These results allow proposing training and orientation to the families using technological means. In conclusion, the guidance finds in this situation a new field of development and growth in this liquid society.

Key Words: mobile devices, families, parental training, parental guidance, media literacy.

\section{Introducción}

La sociedad del siglo XXI podrá definirse en un futuro por factores políticos centrados en la existencia de conflictos locales, la multiplicación de focos terroristas y el descontrol de los Estados 
sobre lo que ocurre en el ciberespacio; por aspectos socioeconómicos que giran en torno a la desigualdad en el crecimiento de los países, a un modelo económico insostenible para el planeta, a la globalización de la economía y al uso de la tecnología en casi todas las actividades humanas; por condicionantes socioculturales focalizados en el avance científico, en la existencia de amplias redes de comunicación, en la omnipresencia de los medios de comunicación a través de diferentes dispositivos móviles, en la accesibilidad al conocimiento, pero también en la manipulación ideológica y el desarrollo de un pensamiento único que pone en riesgo la identidad cultural de diferentes pueblos y en el cambio estructural que sufre la familia, entre otras cuestiones.

La familia, como institución básica de la sociedad, no es ajena a estas transformaciones y en su funcionamiento la tecnología también ha ocupado un lugar destacado. En este sentido, el Informe Anual del Ministerio de Economía y Empresa (2019) basado en los datos del Observatorio Nacional de las Telecomunicaciones y de la Sociedad de la Información (ONTSI, 2019) señala que en octubre de 2018 el $98 \%$ de los hogares españoles se encontraban equipados con telefonía móvil, el $82.2 \%$ estaban conectados a Internet mediante diferentes dispositivos electrónicos (Smartphone, un 88.7\%; ordenador portátil, un 53.9\%; ordenador de sobremesa, $45.8 \%$; tableta, un $35.6 \%$, Smart TV, un $16 \%$ y otros dispositivos un $7 \%$ ). Asimismo, el estudio sobre Equipamiento y uso de tecnologías de información y comunicación en los hogares del Instituto Nacional de Estadística (INE, 2019) muestra que el $66 \%$ de niños comprendidos entre los 10 y los 15 años de edad cuentan con un teléfono móvil propio. También se evidencia que niños de 2 y 3 años tienen acceso al Smartphone de sus padres (Cánovas, 2014) o a otros dispositivos móviles como Tablets (Alfaro et al., 2015; Berríos, Buzarrais, y Garcés, 2015).

No resulta extraño, pues, que el hogar se configure como contexto de alfabetización digital en el que los menores aprenden a reconocer el papel de la tecnología (Marsh, Hannon, Lewis, y Ritchie, 2017; Plowman, Stevenson, Stephen, y McPake, 2012), gracias a experiencias ofrecidas por sus progenitores y que convierten a los dispositivos móviles en instrumentos prácticamente imprescindibles en su vida diaria (Pfetsch, 2018).

El Estudio de uso y actitudes de consumo de contenidos digitales (Ministerio de Energía, Turismo y Agenda Digital, 2017) mostraba la inquietud existente sobre diferentes aspectos vinculados a los teléfonos móviles inteligentes en los menores: generación de situaciones antisociales y de aislamiento (75.8\%), dependencia tecnológica $(71.1 \%)$, pérdida de reflexión y de valor de los contenidos por un exceso de información (más del $50 \%$ ) y problemas de privacidad e intimidad (67.1\%).

Según Caro y Plaza (2016), estas cuestiones constituyen "un reto para la Orientación psicopedagógica de los jóvenes afectados" (p.101), afirmación que podríamos completar con un segundo término "y de sus familias", puesto que si las familias, a juicio de Triana (2018, p. 344), han de afrontar situaciones que pueden "desbordar sus capacidades y grados de seguridad ante el ejercicio parental", como consecuencia de la escasez de tiempo para conciliar vida familiar y laboral, la presión económica, el desconocimiento del desarrollo evolutivo de sus hijos e hijas, la falta de estrategias parentales que posibiliten una educación de sus hijos, la atención a otros miembros de la familia extensa como los abuelos o a miembros de la familia que manifiestan necesidades educativas especiales o las nuevas modalidades familiares, la llegada de nuevos componentes tecnológicos a las familias puede incrementar este sentimiento de incompetencia y, por tanto, una mayor necesidad de apoyo y de orientación.

Aunque diferentes investigaciones revelan que las familias suelen tener dificultad para acceder a recursos tecnológicos (Bouffard, 2008; Heath et al., 2015; Segura y Martínez, 2011), muchas otras mantienen que el problema no se encuentra en el acceso, sino en la ausencia de conocimientos y habilidades por parte de las mismas (Sánchez-Garrote y Cortada-Puyol, 2015), que, incluso, llegan a manifestar sus propias limitaciones (Ballesta y Cerezo, 2011; GonzálezFernández, Ramírez-García, y Salcines-Talledo, 2018; Segura y Martínez, 2011). 
La digitalización del hogar reta a las familias a gestionar el consumo interno de contenidos audiovisuales, pues como expresan López-Sánchez y García del Castillo (2017), cada vez más, los menores usan "dispositivos móviles con acceso a Internet fuera del control de los padres o adultos de referencia" (p.108), a pesar de que en los centros educativos se educa para el desarrollo curricular de la competencia digital, para un uso responsable de las Tecnologías de la Información y Comunicación (TIC), para gestionar el conocimiento a través de las Tecnologías del Aprendizaje y del Conocimiento (TAC), así como para el acercamiento a nuevos fenómenos en el universo digital: la participación y la influencia a través de las Tecnologías del Empoderamiento y la Participación (TEP), bien mediante asignaturas y/o materias concretas, bien mediante sesiones de acción tutorial en el ámbito de la prevención y el desarrollo.

El reto no es afrontado por igual en cada familia, las características del modelo familiar y las competencias que desplieguen los progenitores provocarán que los resultados de esta gestión sean totalmente divergentes. En este sentido, Cano y Casado $(2015$, p.17) señalan que "muchos padres tienen dificultades para educar a sus hijos porque 'no saben', 'no pueden' o 'no quieren'”. La experiencia profesional nos permite recordar sesiones de tutoría con las familias en las que estas manifiestan sus limitaciones, su incapacidad para hacer frente a estas situaciones y la petición de ayuda o "recetas" que faciliten su labor de padres y madres. Según Forner (2006, p. 136):

Una buena relación de los padres con la escuela en el marco de la orientación tutorial puede ayudar a muchas familias a encauzar sus prácticas educativas, a asumir sus responsabilidades, a colaborar en la orientación de sus hijos y, además, a confiar en el sistema.

Aquellas familias que han percibido la trascendencia de sus funciones como padres y madres y su repercusión en el desarrollo de los menores se encontrarán en mejor disposición de dar respuesta a las exigencias de la sociedad digital. Sin embargo, existen otras que requieren de una "ayuda" suplementaria en el ejercicio de su parentalidad. Desde una visión holística de la Orientación como disciplina proactiva, tal y como la considera Vera (2011), esta puede convertirse en el soporte de las familias, ya que la Orientación se centra precisamente en ayudar a las personas a utilizar sus propios recursos personales y contextuales para afrontar diferentes tipos de situaciones. Asimismo, como manifiestan Domínguez, Álvarez, y López (2011), los profesionales de la Orientación educativa se posicionan como las figuras idóneas para realizar un acompañamiento, ayuda y asesoramiento a las personas a lo largo de su vida.

Las familias no son las únicas instituciones que han manifestado esta inquietud por el ejercicio de una parentalidad acorde con las necesidades y demandas de sus hijos. Diversos informes publicados por parte la Unión Europea (Comisión Europea, 2011; Comisión Europea, 2012) y la Organización para la Cooperación y el Desarrollo Económico (OCDE, 2011) reconocen la trascendencia del rol parental y familiar en el desarrollo relacional y cognitivo de los menores. Por su parte, el Consejo de Europa ha mostrado esta preocupación e interés por las familias mediante la firma del Convenio para la Protección de los Derechos Humanos y las Libertades Fundamentales, la Carta Social Europea y la Carta Social Europea Revisada, así como la Estrategia de Cohesión Social Revisada.

La parentalidad se define, según la Recomendación del Comité de Ministros de la Unión Europea (Consejo de Europa, 2006), como una etapa de la vida familiar que tiene lugar en un contexto preciso y que genera vínculos entre los miembros de la familia. Este foco de interés sobre la familia cuenta con una larga tradición en la política europea. No obstante, a partir de la publicación de esta Recomendación surgirá el concepto de parentalidad positiva y se expresará la necesidad de reconocimiento por parte de los Estados de la "importancia de la responsabilidad parental y la necesidad de que los padres tengan suficientes apoyos para cumplir con sus responsabilidades en la educación de sus hijos" (p.1). 
La educación de los hijos, por tanto, se ha convertido en un objetivo no solo de las familias, sino también de las propias instituciones comunitarias e internacionales, que contemplan y reconocen la dificultad de la institución familiar de hacer frente a una configuración de una nueva sociedad: líquida (Bauman, 2003), de la información y del conocimiento (Burch, 2005), sociedad red (Castells, 2006), de la ignorancia (Brey, Innerarity y Mayos, 2009), de la incertidumbre (Suárez, Bajoit y Zubillaga, 2013) o de la inmediatez.

Recientes investigaciones proponen la necesidad de impulsar el desarrollo de una parentalidad positiva antes los dispositivos móviles (Ramírez-García, González-Fernández, y Salcines-Talledo, 2018a; Ramírez-García, González-Fernández, y Salcines-Talledo, 2018b; Ramírez-García, González-Fernández, y Salcines-Talledo, 2018c)).

Para que las familias sean capaces de crecer y evolucionar en el marco de una parentalidad positiva, es necesario que cuenten con diferentes apoyos -familiares, económicos, sociales, asistenciales, etc.- "pero también de formación" (Triana, 2018, p. 344).

De acuerdo con Pérez-Bóveda y Yániz (2015, p.105), el enfoque formativo propuesto por el denominado Life Long Learning "plantea que el aprendizaje es un proceso inacabado que se reorganiza a lo largo de distintas etapas del ciclo vital". Así, la educación familiar se enmarcaría dentro de esta tendencia generalizada desde las últimas décadas del siglo XXI y se centraría en la orientación para hacer frente a los retos de la etapa parental y el desarrollo familiar como parte del ciclo vital de una persona". En este sentido, Romero y Montilla (2015) subrayan la idea de que "la orientación educativa debe evolucionar y producir nuevas estrategias acordes a las nuevas tendencias sociales" (p. 80). De este modo, la orientación familiar, entendida como proceso de ayuda prestado a los miembros de la familia en las diferentes etapas de su ciclo vital (FernándezHawrylak, 2002), abre un nuevo campo de acción para los profesionales de la Orientación, especialmente, si este se sitúa en el ámbito de los dispositivos móviles y su conexión a Internet. En esta dirección apuntaba Benavente (2003) cuando afirmaba que la evolución de la orientación psicopedagógica y sus nuevas funciones se encontraba ligada nítidamente a "la prevención y el tratamiento de los problemas suscitados por la falta de socialización de las nuevas generaciones y la aparición de nuevas patologías generadas por la soledad, la "tele-adicción" y las "ciberdependencias" (p. 46).

Según Ríos (1994) y Caro y Plaza (2016) se pueden identificar tres niveles en la Orientación familiar: 1) Nivel educativo o preventivo, que se dedica a la formación de padres e hijos; 2) Nivel de asesoramiento, que adopta una función compensatoria en las distintas etapas del ciclo vital de las familias; 3) Nivel terapéutico, que se centra en la propuesta de nuevas pautas para modificar las pautas de comportamiento familiar.

El enfoque sistémico es el considerado más adecuado para abordar acciones con las familias (Ríos, 1999; Cervel, 2004; Caro y Plaza, 2016). Una propuesta que hacen Caro y Plaza (2016) para el nivel terapéutico es basarse en tres aspectos básicos: participación, implicación emocional y consenso normativo. Sin embargo, a juicio de Triana (2018), en la intervención con las familias ha de primar más la labor preventiva que la simplemente asistencial, la movilización de capacidades y potencialidades parentales frente a la focalización en los déficits familiares.

El nivel de orientación familiar en el que se posicionen las diferentes intervenciones dependerá del objetivo de cada una de ellas, de los factores que inciden en la familia o del contenido de la intervención, entre otros.

Tradicionalmente, los programas de formación parental se han desarrollado desde los Servicios Públicos, algunos de los que han tenido una mayor repercusión en el ámbito internacional por las evidencias que han demostrado son los siguientes: Systematic Training for Effective Parenting (STEP), Active Parenting Now (Popkin, 2008) o Triple P: Positive Parenting Program (Sanders, 2008), entre otros. 
En el caso de España, algunos de los programas que han destacado también son: Escuela de Padres ECCA, Aprender en la vida cotidiana (Máiquez, Rodrigo, Capote, y Vermaes, 2000), Desarrollo de competencias emocionales, educativas y parentales (Martínez, 2009), Programa EDUCA. Escuela de padres. Educación positiva para enseñar a tus hijos (Díaz-Sibaja, Trujillo, Perís-Mencheta, y Pérez-Portas), Educar en familia (Martínez, 2011), Proyecto ROMA (Soler, Gómez, y Sánchez, 2018) y Educar en Positivo (Torres, Suárez, y Rodrigo, 2014).

La mayor parte de estos programas no abordan aspectos vinculados con las nuevas necesidades familiares sobre los medios de comunicación y los dispositivos móviles, a pesar de que esta temática se está convirtiendo en un foco de preocupación para familias y docentes como han demostrado investigaciones como Gradaílle, Varela, y De Valenzuela (2016) por los riesgos que han proliferado: acceso de pederastas, abuso de menores (Catalina, López de Ayala, y García, 2014; Garmendia, Garitaonandia, Martínez, y Casado, 2011; Livingstone, Haddon, Görzig, y Ólafsson, 2010), extorsión con fotografías o vídeos (Gutiérrez, Vega y Rendón, 2013), ciberagresiones (Del Rey, Mora-Merchán, Casas, Ortega-Ruiz, y Elipe, 2018) o consumo de pornografía (Romero-Hernández, 2017). Todas estas y otras situaciones vinculadas a la relación con los dispositivos móviles se están convirtiendo en un problema de salud pública, aunque el DSM-V (APA, 2013) haya rechazado la inclusión de las adicciones sin sustancia como una enfermedad o un trastorno.

En consonancia con este marco conceptual, el objetivo de este trabajo es identificar las necesidades formativas de las familias españolas en relación al uso de los Smartphones y Tablets en el hogar.

Los interrogantes que se plantean en la investigación son: ¿existe una predisposición de las familias españolas a recibir formación sobre el uso de los dispositivos móviles?, ¿qué variables del perfil sociodemográfico de las familias españolas inciden en dicha predisposición? ¿cuáles son los factores sociodemográficos que condicionan la temática y formato de la formación parental?

\section{Método}

El diseño metodológico de este estudio presenta un carácter cuantitativo, no experimental (Hernández, Fernández, y Baptista, 2014) y descriptivo transversal (Sáez-López, 2017).

\section{Muestra}

La muestra de esta investigación está compuesta por 1082 integrantes de familias, pertenecientes a las 17 autonomías y las 2 ciudades autónomas (23.6\% hombres y $76.4 \%$ mujeres).

De acuerdo con el criterio del muestreo aleatorio simple para muestras infinitas, poblaciones superiores a 100.000 personas (como es este caso, según datos del INE, 2019), la muestra es estadísticamente significativa en un nivel de confianza del $99 \%$ y un error del $4 \%$. El perfil 
sociodemográfico vinculado a los resultados significativos hallados en la investigación se expresa en la tabla 1.

\section{Tabla 1}

Perfil sociodemográfico de la muestra

\begin{tabular}{|c|c|}
\hline Variables & $\%$ \\
\hline Comunidad Autónoma & $\begin{array}{l}\text { Andalucía }=11.9 \\
\text { Aragón }=2.4 \\
\text { Asturias }=1.5 \\
\text { Cantabria }=27.8 \\
\text { Castilla La Mancha }=1.4 \\
\text { Castilla Leó }=7.9 \\
\text { Cataluña }=3.1 \\
\text { Comunidad Valenciana }=1.7 \\
\text { Extremadura }=1.9 \\
\text { Galicia }=2.1 \\
\text { Islas Baleares }=0.4 \\
\text { Islas Canarias }=8.4 \\
\text { La Rioja }=2.2 \\
\text { Madrid }=10.5 \\
\text { Navarra }=0.3 \\
\text { País Vasco }=14.1 \\
\text { Ciudades autónomas de Ceuta y Melilla }=0.3\end{array}$ \\
\hline Tener hijos & $\begin{array}{l}\text { Sí }=90.5 \\
\mathrm{No}=9.5\end{array}$ \\
\hline $\begin{array}{l}\text { Disponibilidad de prensa y/o } \\
\text { revistas especializadas }\end{array}$ & $\begin{array}{l}\text { Sí=50.5 } \\
\text { No }=49.5\end{array}$ \\
\hline Uso de la Tablet & $\begin{array}{l}\text { UMR }=67.6 \\
\text { UME }=19.4 \\
\text { No dispone }=10 \\
\text { No sabe/ no contesta }=3\end{array}$ \\
\hline Uso de e-Book & $\begin{array}{l}\text { UMR }=38.7 \\
\text { UME }=6.4 \\
\text { No dispone del dispositivo }=40 \\
\text { No sabe/ no contesta }=14.9\end{array}$ \\
\hline Uso del Smartphone & $\begin{array}{l}\text { UMR }=66.2 \\
\text { UME }=27.4 \\
\text { No dispone }=2.6 \\
\text { No sabe/ no contesta }=3.81\end{array}$ \\
\hline $\begin{array}{l}\text { Utilización de los } \\
\text { dispositivos para adquirir } \\
\text { conocimientos }\end{array}$ & $\begin{array}{l}\text { Tablet }=50 \\
\text { Smartphone }=38.1 \\
\text { No sabe/no contesta }=11.9\end{array}$ \\
\hline
\end{tabular}

$\mathrm{UMR}=$ Uso mayoritariamente recreativo; UME=Uso mayoritariamente educativo.

Fuente: Elaboración propia. 


\section{Instrumentos}

El instrumento empleado en el estudio ha sido diseñado ad hoc y se estructura del siguiente modo: datos identificativos, conocimientos generales, conocimiento sobre los dispositivos móviles, comunicación familiar, rutinas y hábitos, normativa familiar, mediación familiar y necesidades formativas. Este instrumento fue sometido a un proceso de validación y fiabilidad, tal y como se publicó en (Salcines-Talledo, Ramírez-García y González-Fernández, 2018), mostrando una fiabilidad de las distintas escalas comprendida entre .709 y .931.

La dimensión "necesidades formativas" se analizará en este trabajo y está compuesta por 12 ítems relativos a predisposición de las familias a la formación, centros de interés de la formación y modalidad formativa (véase tabla 2). Las opciones de respuesta de los mismos era Sí/No.

\section{Tabla 2}

Ítems de la dimensión "necesidades formativas" del cuestionario

\begin{tabular}{ll}
\hline \multicolumn{1}{c}{ Dimensiones } & \multicolumn{1}{c}{ Ítems } \\
\hline $\begin{array}{l}\text { Predisposición a } \\
\text { la formación }\end{array}$ & $\begin{array}{l}\text { Le gustaría recibir una formación para ampliar su conocimiento sobre el uso de los } \\
\text { dispositivos móviles en el contexto familiar }\end{array}$ \\
$\begin{array}{ll}\text { Centros de } \\
\text { interés de la } \\
\text { formación }\end{array}$ & $\begin{array}{l}\text { Sobre normativa y regulación de los dispositivos y contenidos móviles en la familia } \\
\text { Sobre estrategias de seguimiento del uso de los dispositivos móviles en la familia }\end{array}$ \\
& $\begin{array}{l}\text { Sobre recursos educativos útiles para los dispositivos móviles } \\
\text { Sobre buenas prácticas familiares en relación al uso de dispositivos móviles }\end{array}$ \\
& Sobre otras temáticas \\
Modalidades & $\begin{array}{l}\text { Audios de no más de 3 minutos } \\
\text { formativas }\end{array}$ \\
& $\begin{array}{l}\text { Audiovisuales de no más de 3 minutos } \\
\text { Documentos escritos }\end{array}$ \\
& $\begin{array}{l}\text { Curso presencial } \\
\text { Curso online }\end{array}$ \\
& Otras modalidades
\end{tabular}

Fuente: Elaboración propia.

\section{Procedimiento}

La encuesta fue suministrada a las familias españolas en dos fases. La primera mediante la difusión en grupos de WhatsApp a través del Smartphone (entre los meses de noviembre y diciembre de 2017), junto al enlace al cuestionario (a través de Limesurvey), se envió un mensaje que garantizaba la protección de datos de los participantes, la posibilidad de no responder y/o difundir el mensaje, así como de abandonar el cuestionario en el momento en que desearan. La segunda utilizando el correo electrónico para contactar con asociaciones de padres y madres de alumnos de colegios e institutos de todas las comunidades autónomas de España que disponían de página web y en ella tenían consignado el correo electrónico de la misma (entre los meses de febrero y abril de 2018). 
En todo momento se llevó a cabo un procedimiento ético y seguro de acuerdo con la Declaración Nacional sobre Integridad Científica (COSCE, CRUE, y CSIC, 2015), pudiéndose considerar esta investigación como una Investigación e Innovación Responsable (García-Marzá, Fernández-Beltrán, y Sanahuja, 2017).

Los datos obtenidos se analizaron con el programa estadístico SPSS (v.22). El análisis descriptivo de las variables que conforman el perfil sociodemográfico de la muestra participante permitió caracterizar dicha muestra. Por su parte, el análisis de contingencia posibilitó determinar la asociación entre diferentes variables.

\section{Resultados}

Los porcentajes de respuesta obtenidos en los diferentes ítems que integraban la dimensión "necesidades formativas" se recogen en la Tabla 3.

Tabla 3

Porcentajes de respuesta en la dimensión "necesidades formativas"

\begin{tabular}{|c|c|c|}
\hline Dimensiones & Ítems & $\%$ \\
\hline $\begin{array}{l}\text { Predisposición } \\
\text { a la formación }\end{array}$ & $\begin{array}{l}\text { Le gustaría recibir una formación para ampliar su } \\
\text { conocimiento sobre el uso de los dispositivos móviles en el } \\
\text { contexto familiar }\end{array}$ & $\begin{array}{l}\text { Sí }=55.7 \\
\text { No }=37.2 \\
\text { No sabe/no contesta }=7.2\end{array}$ \\
\hline \multirow{5}{*}{$\begin{array}{l}\text { Centros de } \\
\text { interés de la } \\
\text { formación }\end{array}$} & $\begin{array}{l}\text { Sobre normativa y regulación de los dispositivos y contenidos } \\
\text { móviles en la familia }\end{array}$ & $\begin{array}{l}\mathrm{Sí}=46.1 \\
\mathrm{No}=53.9\end{array}$ \\
\hline & $\begin{array}{l}\text { Sobre estrategias de seguimiento del uso de los dispositivos } \\
\text { móviles en la familia }\end{array}$ & $\begin{array}{l}\text { Sí=69.5 } \\
\text { No }=30.5\end{array}$ \\
\hline & $\begin{array}{l}\text { Sobre recursos educativos útiles para los dispositivos } \\
\text { móviles }\end{array}$ & $\begin{array}{l}\text { Sí=73.9 } \\
\text { No=26.1 }\end{array}$ \\
\hline & $\begin{array}{l}\text { Sobre buenas prácticas familiares en relación al uso de } \\
\text { dispositivos móviles }\end{array}$ & $\begin{array}{l}\text { Sí=75.7 } \\
\text { No=24.3 }\end{array}$ \\
\hline & Sobre otras temáticas & $\begin{array}{l}\text { Sí=6 } \\
\text { No=94 }\end{array}$ \\
\hline \multirow[t]{6}{*}{$\begin{array}{l}\text { Modalidades } \\
\text { formativas }\end{array}$} & Audios de no más de 3 minutos & $\begin{array}{l}\text { Sí }=14.7 \\
\text { No }=85.3\end{array}$ \\
\hline & Audiovisuales de no más de 3 minutos & $\begin{array}{l}\text { Sí }=51.7 \\
\text { No }=48.3\end{array}$ \\
\hline & Documentos escritos & $\begin{array}{l}\text { Sí }=47.7 \\
\mathrm{No}=52.3\end{array}$ \\
\hline & Curso presencial & $\begin{array}{l}\mathrm{Sí}=18.5 \\
\mathrm{No}=81.5\end{array}$ \\
\hline & Curso online & $\begin{array}{l}\text { Sí=34 } \\
\text { No=66 }\end{array}$ \\
\hline & Otras modalidades & $\begin{array}{l}\text { Sí=3.5 } \\
\text { No=96.5 }\end{array}$ \\
\hline
\end{tabular}

Fuente: Elaboración propia. 
Al preguntar a las familias si les gustaría recibir formación para ampliar su conocimiento sobre el uso de los dispositivos móviles en el contexto familiar, un $55.7 \%$ respondió en que sí aceptarían recibirla.

En cuanto a los centros de interés o temáticas posibles sobre los que versaría la formación, se observa que las familias están predispuestas a una formación centrada en buenas prácticas familiares en relación con el uso de los dispositivos móviles (75.7\%), seguida de formación sobre recursos educativos para los dispositivos (73.9\%), estrategias de seguimiento del uso de los dispositivos móviles (69.5\%) y otras temáticas (6\%) (abuso del uso de estas nuevas tecnologías, peligro de las TIC, sentimientos de culpabilidad al no controlar el uso, etc.).

Respecto a las modalidades formativas, destaca en primer lugar un formato basado en audiovisuales de no más de 3 minutos (57.7\%), seguido de documentos escritos (47.7\%), curso online (34\%), curso presencial (18.5\%), audios de no más de 3 minutos $(14.7 \%)$ y, finalmente, otras modalidades (3.5\%).

Algunas de las propuestas que expresan las familias sobre otras modalidades son: reuniones en los centros educativos, escuelas de padres y madres y charlas de expertos, que bien pudiera tratarse de un orientador.

\section{Tabla 4}

Análisis de contingencia

\begin{tabular}{|c|c|c|c|c|}
\hline $\begin{array}{c}\text { Variables } \\
\text { independientes }\end{array}$ & Variables dependientes & Valor $\chi^{2}$ & gl & $\begin{array}{c}\text { Significación } \\
\text { asintótica bilateral }\end{array}$ \\
\hline $\begin{array}{l}\text { Comunidades } \\
\text { Autónomas }\end{array}$ & $\begin{array}{l}\text { Reflexión buenas } \\
\text { prácticas }\end{array}$ & 24.280 & 14 & .042 \\
\hline \multirow[t]{2}{*}{ Tener hijos } & $\begin{array}{l}\text { Audiovisuales de no más } \\
\text { de } 3 \text { minutos }\end{array}$ & 3.981 & 1 & .046 \\
\hline & Documentos escritos & 3.877 & 1 & .049 \\
\hline $\begin{array}{l}\text { Disponibilidad de } \\
\text { prensa y/o revistas } \\
\text { especializadas }\end{array}$ & $\begin{array}{l}\text { Le gustaría recibir } \\
\text { información }\end{array}$ & 7.311 & 2 & .026 \\
\hline Uso de la tablet & $\begin{array}{l}\text { Estrategias de } \\
\text { seguimiento del uso de } \\
\text { los dispositivos móviles } \\
\text { en la familia }\end{array}$ & 9.662 & 3 & .022 \\
\hline Uso del Smartphone & $\begin{array}{l}\text { Estrategias de } \\
\text { seguimiento del uso de } \\
\text { los dispositivos móviles } \\
\text { en la familia }\end{array}$ & 7.913 & 3 & .048 \\
\hline Uso del eBook & $\begin{array}{l}\text { Documentos escritos } \\
\text { Curso presencial }\end{array}$ & $\begin{array}{c}8.107 \\
11.201\end{array}$ & $\begin{array}{l}3 \\
3\end{array}$ & $\begin{array}{l}.044 \\
.011\end{array}$ \\
\hline $\begin{array}{l}\text { Utilización de los } \\
\text { dispositivos para } \\
\text { adquirir conocimientos }\end{array}$ & Curso presencial & 7.462 & 2 & .024 \\
\hline
\end{tabular}

Fuente: Elaboración propia. 
Posteriormente, se realizó un análisis de contingencia para comprobar la asociación existente entre diferentes variables. Este análisis partió de la consideración de que el perfil sociodemográfico de las familias podía incidir en la predisposición de las familias a la formación, los centros de interés de esta formación y de las modalidades formativas propuestas. Los resultados que ofrecían significatividad estadística se han recogido en la Tabla 4 . No obstante, el análisis del porcentaje de frecuencias esperadas indicó que había que desestimar la prueba de $\chi^{2}$ para la variable independiente procedencia de las distintas Comunidades Autónomas. Por tanto, solo se puede afirmar que el tener hijos 0 no, la disponibilidad de prensa y/o revistas especializadas en el hogar, el uso de los dispositivos móviles - Tablet, Smartphone y eBook- y el uso de estos dispositivos para adquirir conocimientos condicionan determinados aspectos de las necesidades formativas de las familias.

Ante la pregunta de si se deseaba recibir formación para ampliar el conocimiento sobre los dispositivos móviles, las familias que disponen de prensa y/o revistas especializadas en el hogar manifiestan que desean esa formación en un $49.8 \%$ de los casos, sin embargo, este porcentaje se eleva cuando las familias no cuentan con este tipo de recursos educativos (59\%).

La temática formativa que ha destacado significativamente ha sido la demanda de "estrategias de seguimiento del uso de los dispositivos móviles en la familia". En este sentido, el uso que se le otorga a tres de estos dispositivos móviles condiciona dicha demanda. Así, las familias que usan la Tablet mayoritariamente con fines recreativos solicitan formación en este tipo de estrategias en un porcentaje superior (71.6\%) que aquellas que la emplean para cuestiones educativas (69.7\%), no disponen del dispositivo (48.1\%) y no sabe o no contesta al respecto del uso que le dan (48.1\%).

En sentido opuesto sucede en el caso del Smartphone, las necesidades formativas en estrategias de seguimiento es menor en familias que emplean el teléfono móvil inteligente desde una óptica recreativa (65.8\%) que las que mayoritariamente lo usan para cuestiones educativas $(74.5 \%)$ o no disponen del dispositivo $(90 \%)$. Por su parte, las familias que afirman no saber o qué contestar al uso que le atribuyen al Smartphone se sitúan en un 37,5\%.

Los resultados en torno al tipo de uso del eBook muestran que las demandas familiares sobre la formación en estrategias de seguimiento es mayor cuando en el hogar no se dispone del dispositivo $(50,4 \%)$, no saben qué contestar o no desean contestar sobre el uso del dispositivo $(48,9 \%)$, que cuando lo utilizan con fines recreativos $(46.4 \%)$ o educativos (15.8\%).

Tener o no tener hijos se configura como otra variable que es necesario considerar para determinar qué tipo de formato es el que seleccionan las familias como más adecuado para la formación. Así, tanto si se tienen hijos como si no, las familias no desean un tipo de formación basada en este soporte en un $52.1 \%$ y $67.9 \%$ de los casos respectivamente.

Cuando la variable "tener hijos" se asocia a una formación basada en audiovisuales de no más de 3 minutos, las familias con hijos no terminan de decidirse por la modalidad, ya que el 50\% se encuentra tanto a favor como en contra de este formato. En el caso de las familias sin hijos, un $66 \%$ se manifiesta a favor de este tipo de audiovisuales, frente a un $34 \%$ que no lo considera oportuno.

En el caso del uso otorgado al eBook, se ha evidenciado que estas no muestran interés por una formación sustentada en documentos escritos cuando lo emplean para cuestiones educativas $(84.2 \%)$, ni recreativas $(53.6 \%)$, ni cuando no saben o no contestan al respecto $(51.1 \%)$, solo cuando no disponen de este dispositivo manifiestan ligeramente una preferencia por la formación a través de documentos escritos (50.4\%). 
Cuando este uso se asocia a una modalidad formativa basada en la asistencia a cursos presenciales, las familias que emplean el eBook mayoritariamente para aspectos educativos se posicionan totalmente en contra de esta asistencia (100\%), cuando lo usan de manera recreativa piensan que esta modalidad formativa no es la más adecuada el $85.5 \%$ de los casos. En un porcentaje similar se sitúan las familias que no disponen de dicho dispositivo (80.9\%). A las familias que no saben o no contestan sobre el uso del eBook no les convence esta modalidad formativa en el $68.1 \%$ de los casos.

Finalmente, cuando las familias discriminan entre los dispositivos móviles para adquirir conocimientos, expresan que no desean cursos de carácter presencial en un $83,3 \%$ y $84.4 \%$ tanto si utilizan la Tablet como el Smartphone respectivamente para acceder al conocimiento.

\section{Discusión y Conclusiones ${ }^{3}$}

El primer interrogante definido en este estudio giraba en torno a determinar si existía una predisposición de las familias españolas a recibir formación sobre el uso los dispositivos móviles en el contexto familiar. A nuestro juicio, el interés de las familias por una formación en este ámbito se cifra en un porcentaje inferior al esperado, a pesar de que como afirman Fernández-Montalvo, Peñalva, y Irazabal (2015), las alteraciones que estos dispositivos electrónicos ocasionan en la dinámica familiar se debe fundamentalmente a una falta de información y formación de los progenitores sobre el universo digital.

En esta dirección, Terras y Ramsay (2016) evidencian que las familias están preocupadas por el uso y el consumo de contenidos digitales que realizan los menores a través de la conexión a Internet mediante Smartphones y Tablets, pero que si estas inquietudes se quieren abordar adecuadamente, se exige un análisis detallado de los factores contextuales, demográficos y psicológicos que, de manera integrada, inciden en estas cuestiones. De igual forma, Livingstone, Cagiltay, y Ólafsson (2015) manifiestan que existe una interacción compleja de factores sociodemográficos (padres/familia, nivel cultural, sociedad y país) que influyen en esta relación con los medios y las demandas en relación a los mismos.

Por ello, este estudio ha realizado un gran esfuerzo por recabar el mayor número posible de variables que pudieran condicionar o influir en este uso y, por tanto, en la formación necesaria. En este sentido, ha sorprendido comprobar que existen muy pocos factores sociodemográficos que se asocien con las variables que integran la dimensión formación parental. Por tanto, la respuesta al segundo y tercer interrogante de investigación que se han planteado se focaliza solo en determinados factores. Esto implica seguir desarrollando investigaciones que contemplen otra serie de factores más complejos como estilos parentales, desarrollo de modalidades parentales,

\footnotetext{
${ }^{3}$ Los autores muestran su agradecimiento a las siguientes Asociaciones de Madres y Padres de Alumnos: CEIP La Almadraba (Alicante), Los Pinos de Huercal (CEIP Clara Campoamor, Almería), Asociación CEIP Santa María Coronada, Badajoz), Escolaltaca (Barcelona), Pere Vila (Barcelona), Paulo Freire (Burgos), Instituto Conde Diego Porcelos (Burgos), Cecilio Muñoz Fillol del IES Francisco Nieva (Ciudad Real), Juan de la Cosa (CEIP Prácticas, Huelva), CEIP Sancho Ramírez (Huesca), Gonzalo de Berceo (La Rioja), IES Escultor Daniel (Logroño), Carlos V (Madrid), Colegio Artica (Madrid), Colegio Enriqueta Aymer Sagrados Corazones (Madrid), Mijas Pueblo (IES Villa de Mijas, Málaga), Colegio Maestro Ávila (Salamanca), IES Cabrera Pinto (La Laguna, Tenerife), CEIP Narciso Brito (Santa Cruz de Tenerife), Zipifamiliak (Colegio Zipiriñe, Vizcaya), Los Ibones (Colegio Miralbueno, Zaragoza), la Almadraba, EGIBIDE y todas aquellas otras que no registraron su nombre.
} 
competencias parentales y que pudieran ofrecer más explicaciones al fenómeno de la formación de los padres.

Uno de los factores que en este estudio se ha revelado como importante es el nivel cultural de las familias, medido a través de las posibilidades de acceso a diferentes recursos educativos como libros de lectura o prensa y/o revistas especializadas. Se entiende que los hogares con un mayor equipamiento cultural específico, como es la prensa o las revistas especializadas, no demandan tanta formación para ampliar su conocimiento sobre los dispositivos móviles como aquellas otras que carecen de este recurso.

Por otro lado, el uso que las familias españolas otorgan a Smartphones, Tablets y eBook provocan diferencias en la solicitud de formación sobre "estrategias de seguimiento del uso de los dispositivos móviles en la familia". En un principio, se podría pensar que un uso mayoritariamente educativo de estos dispositivos móviles conllevaría una menor demanda formativa en esta temática. Esta situación se produce en el caso de la Tablet y del eBook. Ello podría deberse a las prestaciones diferenciadas de los dispositivos, a las particulares del Smartphone y a una mayor concienciación de los riesgos existentes por parte de las familias que usan el Smartphone con fines educativos. De este modo, como manifiesta (Pfetsch, 2018), si los medios son diferentes, las estrategias que se han de desarrollar también han de ser diferentes.

En cuanto al formato de la formación parental, los resultados han demostrado que factores como tener o no tener hijos, uso del eBook y empleo de los dispositivos móviles para adquirir conocimientos inciden en un rechazo a modalidades formativas basadas en un documento escrito o un curso presencial. El recurso formativo en soporte audiovisual constituye una opción para las familias, pero todavía no marca una tendencia creciente, pese a encontrarnos en una sociedad en la que prima lo audiovisual.

Esta conclusión resulta interesante para el diseño de propuestas formativas para las familias en el marco de una parentalidad positiva centrada en el uso de los dispositivos móviles en el hogar. Según Macià y Garreta (2018), las políticas educativas tienen el compromiso de alfabetizar digitalmente a toda la comunidad, incluidas las familias, por lo que las distintas acciones que se suelen llevar a cabo desde los centros escolares -documentos informativos, talleres, charlas, conferencias, Escuelas de Madres y Padres, etc.- (si bien estas últimas son consideradas por Cano y Casado, 2015) como facilitadoras del desarrollo de prácticas eficaces de orientación educativa), convendría replantearlas y adaptarlas a una nueva realidad digital, como plantea un programa on-line como recurso de apoyo para las familias reconstituidas (Triana, 2018). Para una sociedad líquida, para las nuevas generaciones de padres y madres de sociedad Red o de la información y la comunicación, la formación también ha de redefinirse, quizá en un nuevo concepto de e-parenting.

En este nuevo contexto digital el orientador puede convertirse en un agente de cambio, no solo educativo, sino también social. Sus conocimientos pedagógicos y psicológicos lo ubican en una posición idónea para acometer nuevos proyectos vinculados con la orientación familiar, tanto en los centros educativos como en escenarios no formales. Para Amber y Martos (2017) es necesario redefinir la figura del orientador en los centros educativos y empoderarla, pues a menudo ha sido desvalorizada por las funciones que se le encomendaba. En esta misma dirección apuntan las aportaciones de O'Connor (2018) sobre las políticas que llevan a cabo los centros educativos para que los orientadores desarrollen un papel clave en los mismos.

La formación parental en relación a la gestión del uso de los dispositivos móviles a través de la orientación familiar puede constituir un nuevo contexto para el desarrollo profesional de los orientadores, bien desde el propio centro educativo a través de acciones directas con las familias como las ya mencionadas o indirectas mediante las relaciones que se llevan a cabo entre la familia y los centros educativos en las sesiones de tutoría, o bien en colaboración con los servicios sociales de la comunidad, lo que posibilitaría la apertura del centro educativo al entorno. Nunca más que ahora el trabajo colaborativo entre familia-tutores-orientadores se convierte en una 
necesidad inmediata para abordar las consecuencias que las transformaciones sociales, especialmente las tecnológicas, tienen sobre los hijos-alumnos. Sin embargo, y a riesgo de parecer pesimistas, la predisposición de los progenitores a la formación que se ha detectado en este estudio bien pudiera responder a la tendencia marcada por Cano y Casado (2015), quienes afirman que un número elevado de padres tiene serias dificultades para educar a sus hijos porque "no saben", "no pueden" o "no quieren". En el caso de que los progenitores no sepan cómo educar a sus hijos en su relación con los dispositivos móviles, la formación podría ser una estrategia adecuada para ello. Si las familias no pueden educar a sus vástagos porque no disponen de competencias para ello, la formación y la orientación podría ser el camino. Sin embargo, si las familias no quieren, ni la formación, ni la orientación, ni las políticas públicas podrán revertir esta situación.

Finalmente, la investigación presenta limitaciones al tratarse de un estudio cuantitativo, que ha conllevado la pérdida de respuestas por abandono de los participantes en el transcurso de la encuesta (de 1651 a 1082 participantes). Igualmente, se circunscribe al territorio nacional, limitando el estudio a una visión nacional. Por lo tanto, permite abrir nuevas líneas de investigación, a través de un estudio cualitativo que recoja la voz de las familias y permita profundizar en las problemáticas detectadas. Del mismo modo, se puede ampliar el estudio a un contexto internacional, así como abordar el papel del profesional de la orientación en la formación de las familias, tanto en ámbitos de educación formal como no formal, o las implicaciones prácticas que tendría el trabajo conjunto entre familias y profesionales de la orientación en un desarrollo evolutivo positivo de los menores. Por último, en relación al valor de la presente investigación, cabe señalar que se enmarca en un proyecto de investigación mucho más amplio, la muestra participante es elevada -proporcionando datos ricos y diversos-, el estudio atiende distintas recomendaciones éticas y, por último, la temática abordada es actual, relevante y de interés para la educación, la sociedad y las administraciones, a quiénes les urge potenciar procesos orientadores desde diferentes servicios públicos.

\section{Referencias bibliográficas}

Alfaro, M., Vázquez, M. E., Fierro, A., Herrero, B., Muñoz, M. F., Rodríguez, L., y Grupo de Educación para la Salud de la AEPap (2015). Uso y riesgos de las tecnologías de la información y la comunicación en adolescentes de 13-18 años. Acta Pediátrica Española, 73(6), 146-151. Recuperado de https://goo.gl/2xMH4x

APA (2013). Diagnostic and Statistical Manual of Mental Disorders (V). Arlington, EEUU: American Psychiatric Publishing.

Amber, D., y Martos, M.A. (2017). Ámbitos y funciones de los orientadores para la mejora educativa en Secundaria en contextos retantes. Una mirada cruzada entre orientadores y directivos. Profesorado. Revista de currículum y formación del profesorado, 21(4), 419-437. Recuperado de https://bit.ly/2X94RSy

Ballesta, J., y Cerezo, M.C. (2011). Familia y escuela ante la incorporación de las tecnologías de la Información y la Comunicación. Educación XX1, 14(2), 133-156. http://dx.doi.org/10.5944/educxx1.14.2.248

Bauman, Z. (2003). Modernidad Líquida. México: Fondo de Cultura Económica. 
Benavente, J.A. (2003). Reflexiones sobre el futuro de la orientación psicopedagógica inmersa en una encrucijada sociocultural. Revista Española de Orientación y Psicopedagogía, 1, 41-59. https://doi.org/10.5944/reop.vol.14.num.1.2003.11604

Berríos, LI., Buzarrais, M.R., y Garcés, M.S. (2015). Uso de las TIC y mediación parental percibida por niños en Chile. Comunicar, 45, 161-168. https://dx.doi.org/10.3916/C44-2015-17

Bouffard, S.M. (2008). Tapping into technology: The role of the Internet in family-school communication. Cambridge, MA: Hardvard Family Research Project. Recuperado de http://goo.gl/bGi1ke

Brey, A., Innerarity, D., y Mayos, G. (2009). La Sociedad de la Ignorancia y otros ensayos. Barcelona: Zero Factory, S.L. Recuperado de http://goo.gl/6IYAIV

Burch, S. (2005). Société de l'information et Société de la connaissance. En V. Peugeot y A. Ambrosi (Eds.), Enjeux de mots. Regards multiculturels sur les sociétés de l'information (pp.49-71). CyF éditions.

Cano, R., y Casado, M. (2015). Escuela y familia. Dos pilares fundamentales para unas buenas prácticas de orientación educativa a través de las escuelas de padres. Revista Electrónica Interuniversitaria de Formación del Profesorado, 18(2), 15-27. http://dx.doi.org/10.6018/reifop.18.2.219491

Cánovas, G. (dir.) (2014). Menores de edad y conectividad Móvil en España. Madrid: Protégeles.

Caro, A., y Plaza, J. (2016). Intervención educativa familiar y terapia sistémica en la adicción adolescente a Internet: Fundamentación teórica. Revista Española de Orientación y Psicopedagogía, 27(1), 99-113. https://doi.org/10.5944/reop.vol.27.num.1.2016.17031

Castells, M. (2006). La Sociedad red: una visión global. Madrid: Alianza Editorial.

Catalina, B., López de Ayala, M. C., y García, A. (2014). Los riesgos de los adolescentes en Internet: los menores como actores y víctimas de los peligros de Internet. Revista Latina de Comunicación Social, 69, pp. 462-485. http://dx.doi.org/10.4185/RLCS-2014- 1020

Cervel, M. (2004). Orientación e intervención familiar. Educación y futuro: revista de investigación aplicada y experiencias educativas, 13, 83-100. Recuperado de https://bit.ly/2rDTWCz

Comisión Europea (2011). Tackling early school leaving: A key contribution to the Europe 2020 Agenda. Communication from the Commission to the European Parliament, the Council, the European Economic and Social Committee and the Committee of the Regions. Recuperado de https://goo.gl/7Y9yiy

Comisión Europea (2012). Education and Training for a smart, sustainable and inclusive Europe. Analysis of the implementation of the Strategic Framework for European cooperation in education and training (ET2020) at the European and national levels. Commission Staff Working Document. Recuperado de https://bit.ly/2xIP1Wt

Consejo de Europa (2006). Recomendación Rec (2006)19 del Comité de Ministros a los Estados Miembros sobre políticas de apoyo a la parentalidad positiva. Recuperado de https://goo.gl/2BZ04c

Consejo de Europa (2011). Recommendación CM/Rec 12 of the Committee of Ministers to member states on children's rights and social services friendly to children and families. Recuperado de https://goo.gl/1XNbRn

COSCE, CRUE, y CSIC (2015). Declaración Nacional sobre Integridad Científica. Recuperado de https://bit.ly/3ddlCnA 
Del Rey, R., Mora-Merchán, J.A., Casas, J.A., Ortega-Ruiz, R., y Elipe, P. (2018). Programa "Asegúrate": Efectos en ciberagresión y sus factores de riesgo. Comunicar, 56, 39-48. https://doi.org/10.3916/C56-2018-04

Díaz-Sibaja, M.A., Trujillo, A., Perís-Mencheta, L., y Pérez-Portas, L. (2008). Tratamiento de los problemas de conducta mediante un programa protocolizado de Escuela de Padres. Revista de Psiquiatría y Psicología del Niño y del Adolescente, 8(1), 12-20. Recuperado de https://bit.ly/2Uq2H2Z

Domínguez, G., Álvarez, F., y López, E. (2011). Orientación Educativa y Tecnologías de la Información y la Comunicación. Nuevas respuestas para nuevas realidades. Sevilla: MAD.

Fernández-Montalvo, J., Peñalva, A., y Irazabal, I. (2015). Hábitos de uso y conductas de riesgo en Internet en la preadolescencia. Comunicar, 44, 113-120. https://doi.org/10.3916/C44-201512.

Forner, A. (2006). Familia-escuela. Entradas y salidas del Sistema Educativo. Momentos clave de la tutoría. En M. Álvarez (Ed.), La acción tutorial: su concepción y su práctica (pp.133-154). Madrid: Ministerio de Educación y Ciencia.

García-Marzá, D., Fernández-Beltrán, F., y Sanahuja, R. (2017). Ética y comunicación en la gestión de la investigación e innovación responsables (RRI). El papel de las unidades de cultura científica y de la innovación (UCC+l). Castellón de la Plana: Universidad Jaume I. http://dx.doi.org/10.6035/Humanitats.2017.52.

Garmendia, M. Garitaonandia, C., Martínez, G., y Casado, M.A. (2011). Riesgos y seguridad en Internet: Los menores españoles en el contexto europeo. Bilbao: Universidad del País Vasco.

Gradaílle, R., Varela, L., y De Valenzuela, Á. (2016). Preocupaciones del profesorado y de las familias sobre los tiempos escolares y de ocio del alumnado de Educación Secundaria Postobligatoria. Revista Interuniversitaria de Formación del Profesorado, 86(30.2), 49-62. Recuperado de https://bit.ly/2VBClaC

González-Fernández, N., Ramírez-García, A. y Salcines-Talledo, I. (2018). Competencia mediática y necesidades de alfabetización audiovisual de docentes y familias españolas. Educación XXI, 21(2), 301-321. doi: https://doi.org/10.5944/educxx1.16384

Gutiérrez, R., Vega, L., y Rendón, A.E. (2013). Usos de la Internet y teléfono celular asociados a situaciones de riesgo de explotación sexual de adolescentes. Salud Mental, 36(1), 41-48. https://doi.org/10.17711/SM.0185-3325.2013.006

Heath, D., Maghrabi, R., y Carr, N. (2015). Implications of Information and Communication Technologies (ICT) for school-home communication. Journal of Information Technology Education: Research, 14, 363-396. Recuperado de http://goo.gl/ngi1qs

Hernández, R., Fernández, C., y Baptista, P. (2014). Metodología de la investigación. Madrid: McGraw-Hill.

Instituto Nacional de Estadística (INE, 2019). Equipamiento y Uso de Tecnologías de Información y Comunicación en los hogares españoles. Recuperado de https://bit.ly/2PYxno3

López-Sánchez, C., y García del Castillo, J.A. (2017). La familia como mediadora ante la brecha digital: repercusión en la autoridad. Revista Colombiana de Ciencias Sociales, 8(1), 108-124. http://dx.doi.org/10.21501/22161201.1928

Macià, M., y Garreta, J. (2018). Accesibilidad y alfabetización digital: barreras para la integración 
de las TIC en la comunicación familia/escuela. Revista de Investigación Educativa, 36(1), 239-257. http://dx.doi.org/10.6018/rie.36.1.290111

Máiquez, M.L., Rodrigo, M.J. Capote, C., y Vermaes, I. (2000). Aprender en la vida cotidiana. Un programa experiencial para padres. Madrid: Editorial Visor.

Marsh, J., Hannon, P., Lewis, M., y Ritchie, L. (2017). Young Children's Initiation into Family Literacy Practices in the Digital Age. Journal of Early Childhood Research, 15(1), 47-60. https://doi.org/10.1177/1476718X15582095

Martínez, R.A. (2009). Programa-Guía para el desarrollo de competencias emocionales, educativas y parentales. Secretaría General Técnica. Ministerio de Sanidad y Política Social. Recuperado de http://goo.gl/3inf2S

(2011). Educar en familia. Guía actualizada para orientadores educativos familiares y para padres y madres. Madrid: Cinca.

Ministerio de Economía y Empresa (2019). La Sociedad en Red. Recuperado de https://bit.ly/2U5HOag

Ministerio de Energía, Turismo y Agenda Digital (2017). Estudio de uso y actitudes de consumo de contenidos digitales. Madrid: Secretaría General Técnica. Recuperado de https://goo.gl/V4Rvmy

O'Connor, P. (2018). How school counselors make a world of difference. Phi Delta Kappan, 99(7), 35-39. Recuperado de https://bit.ly/2052IJ8

Pfetsch, J. (2018). Jugendliche Nutzungdigitaler Medien und elterliche Medienerziehung - Ein Forschungsüberblick. Praxis der Kinderpsychologie und Kinderpsychiatrie, 67(2), 110-133. https://doi.org/10.13109 / prkk.2018.67.2.110

Pérez-Bóveda, A., y Yániz Álvarez de Eulate, C. (2015). Programas de formación parental. Análisis comparativo. Revista Española de Orientación y Psicopedagogía, 26(2), 104-122. https://doi.org/10.5944/reop.vol.26.num.2.2015.15231

Plowman, L., Stephen, C., Stevenson, O., y McPake, J. (2012). Preschool children's learning with technology at home. Computers y Education, 59(1), 30-37. https://doi.org/10.1016/j.compedu.2011.11.014

Popkin, M. H. (2008). Active Parenting of Teens. Leader's Guide. 3er edition. Atlanta: Active Parenting Publishers.

Ramírez-García, A., González-Fernández, N. y Salcines-Talledo, I. (2018a). Vulnerabilidad infantil ante los smartphones: en busca de la competencia parental positiva. Lumina. Revista do Programa de Pós-graduação em Comunicação, 12(1), 68-89. Recuperado de https://bit.ly/2KnG5LP

(2018b). Competencias parentales positivas y Smartphones. Diagnóstico en el contexto familiar. Revista Mediterránea de Comunicación, 9(2), 137-157. Recuperado de https://bit.ly/2D6DvDP

(2018c). Nuevos dispositivos móviles, nuevas responsabilidades. En I. Aguaded y L.M. Romero (coord.). Competencia mediática en medios digitales emergentes (pp. 133-149). Salamanca: Comunicación Social.

Ríos, J. A. (1994). Manual de Orientación y Terapia familiar. Madrid: Instituto de Ciencias del Hombre. 
Ríos, J. A. (1999). ¿Enfermos o familias que hacen enfermar? Revista Española de Orientación y Psicopedagogía, 10(17), 163-178. https://www.redalyc.org/pdf/3382/338246652007.pdf

Romero-Hernández, M. (2017). Technology and child pornography in Colombia, 2013-2015: Interpretation from a victimology approach. Revista Criminalidad, 59(1), 27-47. Recuperado de https://bit.ly/2Uv8OTN

Romero, C., y Montilla, M.V.C. (2015). La utilización de las TIC en la Orientación Educativa: un estudio exploratorio sobre la situación actual de uso y formación entre los profesionales de la orientación. Revista Española de Orientación y Psicopedagogía, 26(3), 78-95. https://doi.org/10.5944/reop.vol.26.num.3.2015.16402

Salcines-Talledo I.; Ramírez-García A. y González-Fernández N. (2018). Smartphones y tablets en familia. Construcción de un instrumento diagnóstico. Aula Abierta, 47 (3), 265 - 272. https://doi.org/10.17811/aula_abierta.47.3.2018.265-272

Sánchez-Garrote, I., y Cortada-Pujol, M. (2015). Recursos digitales en la relación familia y escuela en la etapa 0-3. Cultura y Educación, 27(1), 221-233. http://dx.doi.org $/ 10.1080 / 11356405.2015 .1006851$

Sanders, M.R. (2008). Triple P-Positive Parenting Program as a Public Health Approach to Strengthening Parenting. Journal of Family Psychology, 22(3), 506-517. Recuperado de https://bit.ly/2Ks5XGu

Segura, M.L., y Martínez, M.C. (2011). Análisis del perfil formativo en TIC de padres y madres en relación con su participación en la acción tutorial con la EEPP Sagrada Familia de Linares. Revista Electrónica de Investigación y Docencia, 6, 77-97. Recuperado de http://goo.gl/JdpGkm

Soler, C., Gómez, B., y Sánchez, M.T. (2018). Transformando el contexto familiar. El Proyecto Roma como modelo educativo de mejora. Revista Interuniversitaria de Formación del Profesorado, 92(32.2), 163-177. Recuperado de https://bit.ly/2Kojb7d

Suárez, H. J., Bajoit, G., y Zubillaga, V. (2013). La sociedad de la incertidumbre. México: Universidad Nacional Autónoma de México, Instituto de Investigaciones Sociales.

Terras, M.M., y Ramsay, J. (2016). Family Digital Literacy Practices and Children's Mobile Phone Use. Frontiers in Psychology, 7. http://dx.doi.org/10.3389/fpsyg.2016.01957

Torres, A., Suárez, A., y Rodrigo, M. J. (2014). Educar en Positivo: Primeros resultados y retos de futuro. Revista Iberoamericana de Sistemas, Cibernética e Informática (RISCI), 11(2), 1-13. Recuperado de https://bit.ly/2U8dJok

Triana, B. (2018). Un programa on-line como recurso de apoyo para las familias reconstituidas. International Journal of Developmental and Educational Psychology, 1, 343-350.

Vera, G. (2011). La Orientación como política de estado y el sistema nacional de Orientación. Un avance educativo en prevención, salud mental y progreso socio-económico venezolano. Recuperado de https://bit.ly/350gloz 
Fecha de revisión: 12 marzo 2020

Fecha de aceptación: 30 marzo 2020 